\title{
Numerical evidence for the spin-Peierls state in the frustrated quantum antiferromagnet
}

\author{
P. W. Leung* and Ngar-wing Lam \\ Department of Physics, Hong Kong University of Science and Technology, Clear Water Bay, Hong Kong
}

(Received 1 August 1995)

\begin{abstract}
We study the spin- $\frac{1}{2}$ Heisenberg antiferromagnet with an antiferromagnetic $J_{3}$ (third-nearest-neighbor) interaction on a square lattice. We numerically diagonalize this " $J_{1}-J_{3}$ " model on clusters up to 32 sites and search for ground-state properties as the frustration parameter $J_{3} / J_{1}$ changes. For "larger" $J_{3} / J_{1}$ we find enhancement of the incommensurate spin order, in agreement with various theoretical predictions. But for intermediate $J_{3} / J_{1}$, the low-lying excitation energy spectrum suggests that this incommensurate order is short range. Analysis of the ground-state expectation of the order parameter and the symmetries of the first excited state suggest that the spin-Peierls state is the best candidate for the ground state of the $J_{1}-J_{3}$ model in an intermediate $J_{3} / J_{1}$ region.
\end{abstract}

The phase diagram of the frustrated spin- $\frac{1}{2}$ Heisenberg antiferromagnet has received much interest in recent years. On a square lattice, frustration can be introduced by furtherthan-nearest-neighbor antiferromagnetic couplings. Shortrange interactions up to a distance of two lattice constants have been studied. This is the " $J_{1}-J_{2}-J_{3}$ " model, which is described by the Hamiltonian

$$
\mathscr{H}=J_{1} \sum_{n n} \mathbf{S}_{i} \cdot \mathbf{S}_{j}+J_{2} \sum_{2 n n} \mathbf{S}_{i} \cdot \mathbf{S}_{j}+J_{3} \sum_{3 n n} \mathbf{S}_{i} \cdot \mathbf{S}_{j}
$$

where the sums run over all first, second, and third nearest neighbors, and all $J_{i}>0$. The classical phase diagram of this model is well known to have transition lines between Néel, collinear, and spiral states. ${ }^{1}$ The critical line separating the Néel and spiral states, $J_{1}-2 J_{2}-4 J_{3}=0$, is called the classical critical line (CCL). The quantum phase diagram is less clear. When the frustration is small (at small $J_{2}$ and $J_{3}$ ), the model possesses Néel order. Various analytical studies including linear spin-wave ${ }^{2,3}$ and large- $N$ expansion ${ }^{4}$ have shown that the ground state possesses collinear and spiral (incommensurate) spin order at large $J_{2}$ and $J_{3}$, respectively. The ground state at intermediate $J_{2}$ and $J_{3}$, particularly along the quantum analog of the CCL, is still controversial. While some theories ${ }^{2,3,5}$ predict that frustration and quantum fluctuation destroy the Néel order to form a state without spin order, others ${ }^{4,6}$ predict that quantum fluctuation can stabilize the Néel state along this critical line. Nevertheless, it seems likely that in between the Néel and spiral phases, there exists an intermediate state without spin order. Large- $N$ (Ref. 4) and series ${ }^{1}$ expansions predict that this intermediate state is spontaneously dimerized. On the other hand, spin-wave theory ${ }^{2,3,6}$ predicts that this intermediate state is a spin liquid.

The search for a spin-liquid state in low-dimensional quantum antiferromagnets has long been a fascinating problem. Such a state is most likely to be found in frustrated systems with large quantum fluctuations. Therefore, the region along the quantum analog of the CCL in the $J_{1}-J_{2}-J_{3}$ model is a good place to search for a spin-liquid state, although other kinds of long-range order (such as spin-Peierls order) have been proposed in the same region. Since the location of this critical line is unknown, it is tedious to work with two adjustable parameters $J_{2}$ and $J_{3}$. Most numerical diagonalization studies of the frustrated Heisenberg model have been for $J_{3}=0 .{ }^{7,8}$ A recent study ${ }^{6}$ has suggested that the end of the critical line on the $J_{2}$ axis is a Lifshitz point and thus not representative of the whole critical line. The purpose of this paper is to study this model on the $J_{3}$ axis. In the following, we will take $J_{1}=1$ and $J_{2}=0$.

Using the Lanczos algorithm, we are able to diagonalize this $J_{1}-J_{3}$ model on a 32 -site square cluster. It is obvious that the 16-site lattice is too small to include the $J_{3}$ interaction because each site has only two (instead of four) distinct third-nearest neighbors. Finite-size scaling of the 16- and 32-site results will not be reliable, except perhaps at small $J_{3}$. Any other square lattices between 16- and 32-site cannot accommodate either the incommensurate or spin-Peierls state which we are going to discuss. Consequently, most of our results are based on the 32-site system. Although we are not able to study the finite-size effects systematically, our results are complementary to other theoretical studies and are particularly important in those areas where different theoretical approaches do not agree.

It is well known that for $J_{3}=0$, the ground state of the $J_{1}-J_{3}$ model exhibits long-range Néel order. ${ }^{9}$ Figure 1 shows the finite-size plot of the staggered magnetization $m^{\dagger}$, defined as $m^{\dagger 2}=S(\pi, \pi) / N$ [see Eq. (2)]. The system sizes are $N=16,24,{ }^{10}$ and 32 . The $1 / \sqrt{N}$ dependence is taken from spin-wave theory for the unfrustrated case. ${ }^{11}$ We can see that at $J_{3}=0.3, m^{\dagger}$ extrapolates to a finite value as $N \rightarrow \infty$. But the linear extrapolation fails at $J_{3}=0.35$. Hence we conclude that the Néel order persists at least up to $J_{3}=0.3$ in the thermodynamic limit. To study the spin order as $J_{3}$ increases further, we calculate the static structure factor,

$$
S(\mathbf{q})=\frac{1}{N} \sum_{k l} e^{i \mathbf{q} \cdot\left(\mathbf{R}_{k}-\mathbf{R}_{l}\right)}\left\langle\mathbf{S}_{k} \cdot \mathbf{S}_{l}\right\rangle
$$

Figure 2 shows $S(\mathbf{q})$ for the 32-site square lattice at different $J_{3}$. It clearly shows that as $J_{3}$ increases from zero, the peak shifts from $(\pi, \pi)$ to $(3 \pi / 4,3 \pi / 4)$ at $J_{3} \sim 0.5$, and then to $(\pi / 2, \pi / 2)$ at $J_{3} \sim 0.7$. This suggests that the Néel order vanishes as $J_{3}$ increases and another spin order develops which has ordering vector along the $(1,1)$ direction. If the system 


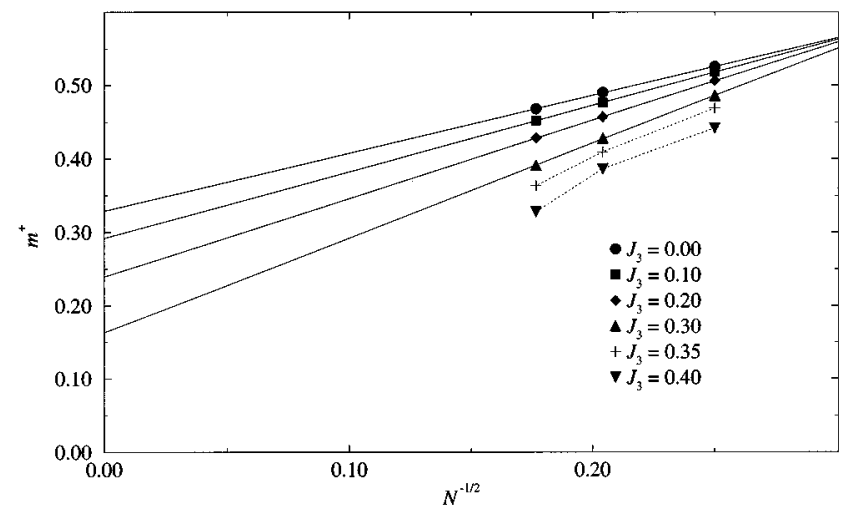

FIG. 1. Finite-size plot of the staggered magnetization $\mathrm{m}^{\dagger}$ at different $J_{3}$. The straight lines are the best fit to the data. The dotted lines are straight lines joining the data points.

possesses incommensurate spin order, as suggested by other theoretical studies, ${ }^{2-4}$ the peak in $S(\mathbf{q})$ should shift continuously from $(\pi, \pi)$ to $(\pi / 2, \pi / 2)$ as $J_{3}$ increases. Due to the discrete nature of the cluster, such a continuous shift along the $(1,1)$ direction is not possible. Nevertheless, Fig. 2 is consistent with this scenario. We also calculate the dynamic structure factor, ${ }^{7} S(\mathbf{q}, \omega)$ (not shown). Sharp low-energy peaks are found at momenta along the $(1,1)$ direction. As $J_{3}$ increases, the lowest-energy peak changes from $(\pi, \pi)$ to $(3 \pi / 4,3 \pi / 4)$ at $J_{3} \sim 0.5$, and then to $(\pi / 2, \pi / 2)$ at $J_{3} \sim 0.7$. This is again consistent with the scenario that as $J_{3}$ increases, Néel order vanishes and the system develops incommensurate spin order.

Another way to check the existence of incommensurate spin order is to calculate the twist correlation function, ${ }^{12}$

$$
\chi_{t}=\left\langle\left|\frac{1}{N} \sum_{\mathbf{r}} \mathbf{S}_{\mathbf{r}} \times\left(\mathbf{S}_{\mathbf{r}+\mathbf{x}}+\mathbf{S}_{\mathbf{r}+\mathbf{y}}\right)\right|^{2}\right\rangle,
$$

where $\mathbf{x}$ and $\mathbf{y}$ are unit vectors. We expect $\chi_{t}$ to be independent of $N$ for large-enough $N$ if the system possesses longrange incommensurate spin order. Figure 3(a) shows $\chi_{t}$ in the 32 -site system at different $J_{3}$. It shows that $\chi_{t}$ is enhanced at $J_{3}$ larger than about 0.4 . This enhancement suggests the existence of incommensurate spin order at large $J_{3}$, which is consistent with the findings from the static and

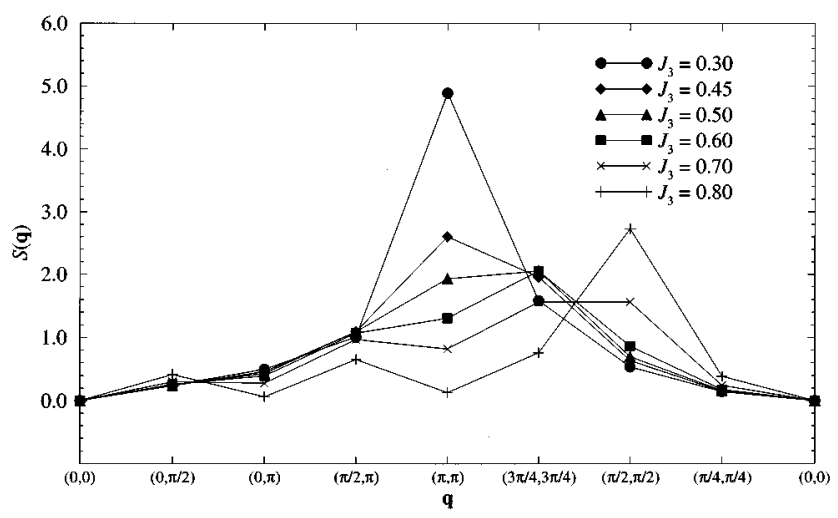

FIG. 2. The static structure factor for the 32-site lattice at different values of $J_{3}$.
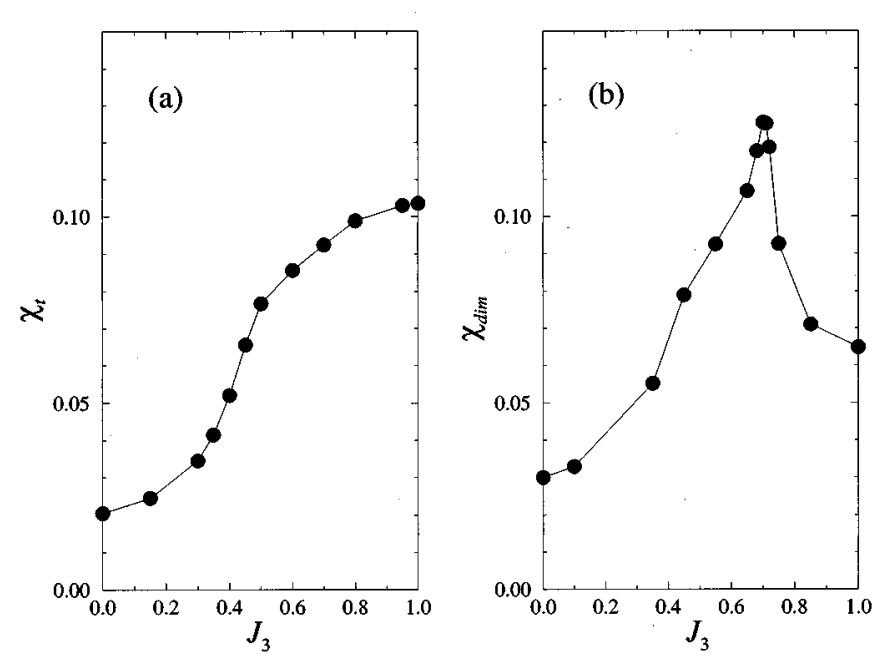

FIG. 3. (a) $\chi_{t}$ and (b) $\chi_{\text {dim }}$ at different $J_{3}$ in the 32-site system.

dynamic structure factors discussed above. However, only spin-wave excitations will show up as peaks in $S(\mathbf{q}, \omega)$. Therefore, it does not exclude the existence of singlet excitations, especially at intermediate $J_{3}$. In particular it is difficult to judge from Fig. 3(a) whether the incommensurate order is long range at intermediate $J_{3}$.

If a quantum system possesses a broken symmetry in the thermodynamic limit, the ground state of the finite system will still be fully symmetric. But there will exist low-lying excited states with the appropriate quantum numbers corresponding to the symmetry which is spontaneously broken in the thermodynamic limit. Consequently, we can use the lowlying energy levels of a finite system to study the possible existence of long-range order. Figure 4 shows the energies of a few low-lying eigenstates in the 32-site system. For $J_{3}$ smaller than about 0.4, the first excited state is a triplet with momentum $(\pi, \pi)$, consistent with the existence of Néel order for small $J_{3}$. We denote this state as $E_{T 1}$. For $J_{3}$ larger than about 0.85 , the first excited state is a triplet with mo-

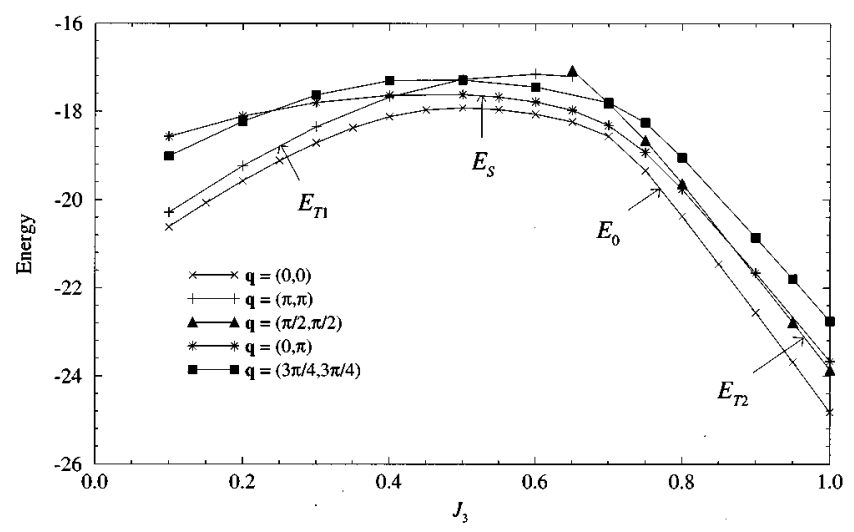

FIG. 4. Lowest-energy states in different momentum sectors at different $J_{3}$ in the 32 -site system. $E_{0}$ is the ground state. $E_{T 1}$ and $E_{T 2}$ are spin triplets while $E_{S}$ is a spin singlet. For the purpose of clarity, lowest-energy states in other momentum sectors and the second-lowest-energy state with momentum $\mathbf{q}=(0,0)$ are not shown. These states have higher energy than the second-lowestenergy states shown in the figure. 
mentum $(\pi / 2, \pi / 2)$. We denote this state as $E_{T 2} . E_{T 1}$ and $E_{T 2}$ are the two spin-wave excitations which show up as low-energy peaks in $S(\mathbf{q}, \omega)$. However, at intermediate $J_{3}$, states with momentum $(3 \pi / 4,3 \pi / 4)$ are never the first excited state. We denote the first excited state in this region as $E_{S}$. It is a twofold degenerate singlet pair, one with momentum $(0, \pi)$ and the other with $(\pi, 0)$. Both are odd under reflection along the direction orthogonal to their momenta. The symmetries of the $E_{S}$ state resemble the columnar dimer state. ${ }^{13}$ In this state, nearest-neighbor spin pairs form singlets (dimers), and these dimers freeze into a columnar order. It is fourfold degenerate, and can form four states with distinct symmetries: two with zero momentum, of which one is fully symmetric while the other is odd under rotation; and two with momenta $(0, \pi)$ and $(\pi, 0)$. The last two have the same symmetries as the degenerate $E_{S}$ state.

Since the finite system always has a first excited state with certain symmetry properties no matter whether the ground state possesses true long-range order, the above study of the low-lying states alone is not sufficient to show the existence of columnar dimer order. Next we study the order parameter for the columnar dimer state, ${ }^{14}$

$$
\theta_{\mathbf{r}}^{\operatorname{dim}}=(-1)^{r_{x}} \mathbf{S}_{\mathbf{r}} \cdot \mathbf{S}_{\mathbf{r}+\mathbf{x}}+i(-1)^{r_{y}} \mathbf{S}_{\mathbf{r}} \cdot \mathbf{S}_{\mathbf{r}+\mathbf{y}} .
$$

In finite-size calculations, we examine the correlation function

$$
\chi_{\mathrm{dim}}=\left\langle\left|\frac{1}{N} \sum_{\mathbf{r}} \theta_{\mathbf{r}}^{\mathrm{dim}}\right|^{2}\right\rangle .
$$

If the ground state has long-range columnar dimer order, $\chi_{\mathrm{dim}} \sim O(1)$ for sufficiently large $N$. Figure 3(b) shows $\chi_{\mathrm{dim}}$ at various $J_{3}$ in the 32 -site system. $\chi_{\mathrm{dim}}$ has a peak at $J_{3} \sim 0.7$, indicating that columnar dimer order is enhanced in this region. We also note that the location of this peak corresponds to the minimum energy gap between $E_{0}$ and $E_{S}$ in Fig. 4.

The spatial variations of the dimer correlation can be demonstrated by calculating the dimer-dimer correlation function ${ }^{15}$ defined as

$$
C_{(i, j)(k, l)}=\left\langle\left(\mathbf{S}_{i} \cdot \mathbf{S}_{j}\right)\left(\mathbf{S}_{k} \cdot \mathbf{S}_{l}\right)\right\rangle-\left\langle\mathbf{S}_{i} \cdot \mathbf{S}_{j}\right\rangle^{2},
$$

where the bracket $(m, n)$ denotes nearest-neighbor sites. A dimer liquid state will display short-range structure in $C_{(i, j)(k, l)}$ but decrease to zero at large dimer separations. On the other hand, a dimer solid, or spin-Peierls state, will continue to show periodic oscillations reflecting the underlying long-range order. $C_{(i, j)(k, l)}$ for all inequivalent dimer pairs of the 32-site lattice evaluated at $J_{3}=0.7$ are tabulated in Table I. Figure 5 is a pictorial representation of $C_{(i, j)(k, l)}$. The reference bond $(i, j)$ is represented by a double line. For all other bonds $(k, l)$, the magnitude of $C_{(i, j)(k, l)}$ is represented by the thickness of the line joining the sites $k$ and $l$. Solid lines represent positive correlation, and broken lines represent negative or anticorrelation. It is clear that nearestneighbor spin pairs tend to form dimers, and the dimers are arranged in a columnar fashion. Around the reference bond, $C_{(i, j)(k, l)}$ shows strong short-range correlations due to the
TABLE I. Dimer-dimer correlation functions for all inequivalent dimer pairs in the 32 -site system at $J_{3}=0.7$. See Fig. 5 for the numbering of the sites. The reference pair is $(19,23)$. The two spin correlation $\left\langle\mathbf{S}_{i} \cdot \mathbf{S}_{j}\right\rangle$ is -0.183819 .

\begin{tabular}{lrrr}
\hline \hline$(k, l)$ & $C_{(19,23)(k, l)}$ & $(k, l)$ & $C_{(19,23)(k, l)}$ \\
\hline$(1,5)$ & 0.055075 & $(19,22)$ & -0.019733 \\
$(1,29)$ & 0.003783 & $(19,23)$ & 0.245620 \\
$(2,29)$ & 0.051208 & $(21,25)$ & 0.003865 \\
$(5,10)$ & -0.051912 & $(21,26)$ & -0.045224 \\
$(10,14)$ & 0.067346 & $(22,26)$ & 0.009617 \\
$(13,18)$ & 0.051260 & $(22,27)$ & 0.104930 \\
$(14,19)$ & -0.071722 & $(25,29)$ & -0.042596 \\
$(17,21)$ & 0.050038 & $(26,29)$ & 0.003208 \\
$(18,21)$ & 0.002528 & $(26,30)$ & 0.067526 \\
$(18,22)$ & -0.054987 & & \\
\hline \hline
\end{tabular}

fact that spin- $\frac{1}{2}$ objects can form dimer with one of their nearest neighbors only. But when we compare $C_{(i, j)(k, l)}$ of the horizontal bonds farther away from the reference bond, they do not vary too much. Except for the trivial short-range order, the dimer-dimer correlations do not decrease appreciably in the largest dimer separation allowed in our system size.

To conclude, our numerical results suggest that Néel order in the $J_{1}-J_{3}$ model is stable up to $J_{3}>0.3$, as compared to $J_{3}=0.25$ in the classical case. This could be the result of the CCL being moved to much larger $J_{2}$ and $J_{3}$ values by quantum fluctuation, ${ }^{6}$ when the Néel state is stabilized (order from disorder) and the spiral state is destabilized along the

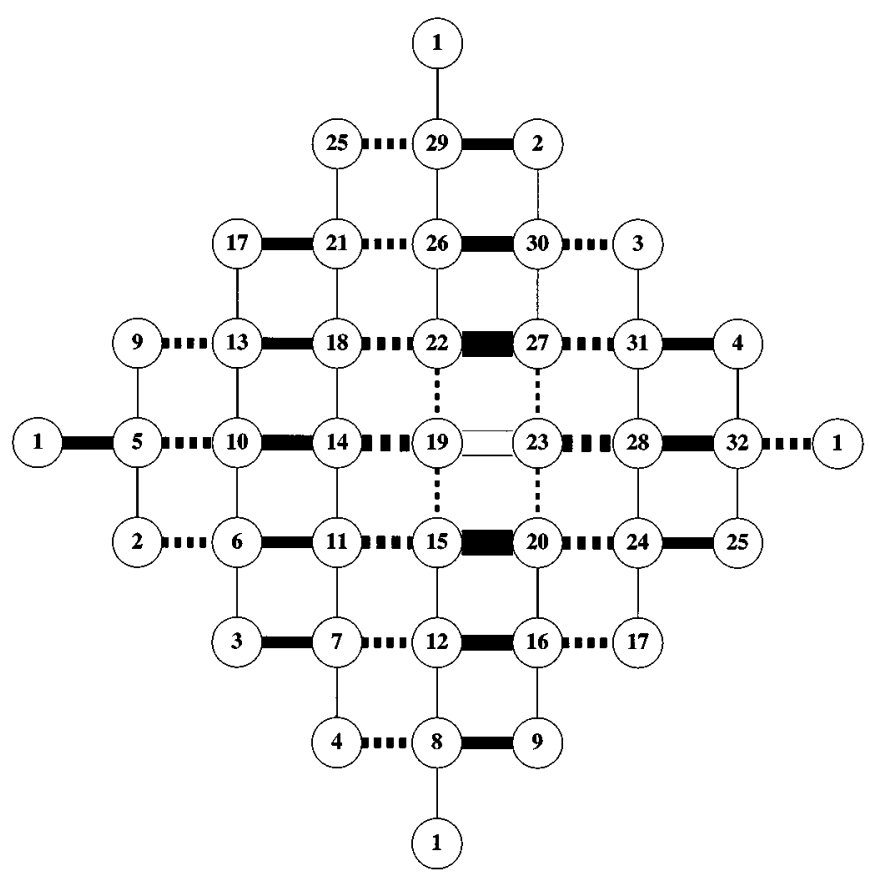

FIG. 5. Dimer-dimer correlation function $C_{(19,23)(k, l)}$ of the 32site system at $J_{3}=0.7$. The reference bond $(19,23)$ is represented by a double line. The magnitude of $C_{(19,23)(k, l)}$ is proportional to the thickness of the line joining the pair of sites $(k, l)$. The solid line means $C_{(19,23)(k, l)}$ is positive, and the broken line means $C_{(19,23)(k, l)}$ is negative. 
critical line. At large $J_{3}$, we believe, as our results suggest, that the model possesses incommensurate spin order. This is consistent with various theoretical studies. Our results further suggest that the model is likely to have a spin-Peierls state between the Néel state (at small $J_{3}$ ) and the incommensurate state (at large $J_{3}$ ), in agreement with some theoretical predictions. ${ }^{1,4,16}$ In particular, large- $N$ expansion ${ }^{4}$ predicted that the dimerized patterns depend on the spin $S$, and our results agree with it for $S=\frac{1}{2}$. We do not find evidence for a spin-liquid state as suggested by spin-wave theory, ${ }^{2,3,6}$ al-

*Electronic address: phleung@usthk.ust.hk

${ }^{1}$ M. P. Gelfand, R. R. P. Singh, and D. A. Huse, Phys. Rev. B 40, 10801 (1989).

${ }^{2}$ P. Chandra and B. Doucot, Phys. Rev. B 38, 9335 (1988); P. Locher, ibid. 41, 2537 (1990); A. V. Chubukov, ibid. 44, 392 (1991).

${ }^{3}$ A. Moreo, E. Dagotto, Th. Jolicoeur, and J. Riera, Phys. Rev. B 42, 6283 (1990).

${ }^{4}$ S. Sachdev and N. Read, Phys. Rev. Lett. 66, 1773 (1991); Int. J. Mod. Phys. B 5, 219 (1991). For a review of this result see S. Sachdev, in Low-Dimensional Quantum Field Theories for Condensed Matter Physicists, edited by L. Yu, S. Lundqvist, and G. Morandi (World Scientific, Singapore, 1995).

${ }^{5}$ L. B. Ioffe and A. I. Larkin, Int. J. Mod. Phys. B 2, 203 (1988).

${ }^{6}$ J. Ferrer, Phys. Rev. B 47, 8769 (1993).

${ }^{7}$ D. Poilblanc, E. Gagliano, S. Bacci, and E. Dagotto, Phys. Rev. B 43, 10970 (1991).

${ }^{8}$ E. Dagotto and A. Moreo, Phys. Rev. Lett. 63, 2148 (1989); F. though our results cannot rule it out unambiguously.

We thank R. J. Gooding, T. K. Ng, N. Read, and S. Sachdev for very useful discussions. This work was supported by Hong Kong RGC Grants No. HKUST619/95P (P.W.L.) and No. HKUST123/92E (N.W.L.). Part of the numerical diagonalizations were performed on an HP workstation cluster at the Center for Computing and Communication Services (CCST) of the Hong Kong University of Science and Technology. Technical support provided by the CCST staff is gratefully acknowledged.

Figueirido, A. Karlhede, S. Kivelson, S. Sondhi, M. Rocek, and D. S. Rokhsar, Phys. Rev. B 41, 4619 (1990); H. J. Schulz and T.

A. L. Ziman, Europhys. Lett. 18, 355 (1992); H. J. Schulz, T. A. L. Ziman, and D. Poilblanc (unpublished). See also Ref. 3.

${ }^{9}$ E. Manousakis, Rev. Mod. Phys. 63, 1 (1991), and references therein.

${ }^{10}$ This 24-site rectangular cluster was first introduced in R. J. Gooding, K. J. E. Vos, and P. W. Leung, Phys. Rev. B 49, 4119 (1994). Although it does not have square symmetry, it has many allowed wave vectors along the $(1,1)$ direction which make it suitable for studying incommensurate spin order.

${ }^{11}$ H. Neuberger and T. A. L. Ziman, Phys. Rev. B 39, 2608 (1989).

${ }^{12}$ P. Chandra, P. Coleman, and A. I. Larkin, Phys. Rev. Lett. 64, 88 (1990).

${ }^{13}$ D. Rokhsar and S. Kivelson, Phys. Rev. Lett. 61, 2376 (1988).

${ }^{14}$ S. Sachdev, Phys. Rev. B 40, 5204 (1989).

${ }^{15}$ P. W. Leung and V. Elser, Phys. Rev. B 47, 5459 (1993).

${ }^{16}$ S. Sachdev and R. N. Bhatt, Phys. Rev. B 41, 9323 (1990). 